\section{Trabeculectomy with ologen in secondary glaucomas following failed trabeculectomy with MMC: comparative study}

HMA El-Saied and MASE Abdelhakim
Department of

Ophthalmology, Kasr Al

Ainy Hospital, Cairo

University, Cairo, Egypt

Correspondence: MASE Abdelhakim, Ophthalmology, Cairo University Hospitals, 11c, Street 199, Apt. \#9, Degla, Maadi, Cairo 11431, Egypt Tel: +0020102592639;

Fax: +0020225165690 .

E-mail:m.amr.salah@

kasralainy.edu.eg

Received: 5 January 2016 Accepted in revised form: 20 April 2016

Published online:

3 June 2016

\begin{abstract}
Purpose We aimed to assess the IOPlowering effect of trabeculectomy with ologen in refractory secondary glaucoma following failed trabeculectomy with mitomycin C (MMC), and to compare its surgical outcome between open angle (SOAG) and angle closure (SACG) cases.

Methods This is a prospective interventional comparative study conducted on 40 eyes (40 patients) with medically uncontrolled secondary glaucoma. Patients were divided into group A: 18 eyes (18 patients) with SOAG, and group B: 22 eyes (22 patients) with SACG. All patients underwent trabeculectomy with insertion of ologen implant. Intraocular pressure (IOP) measurement, SITA standard perimetry (Central 24-2), spectral domain optical coherence tomography (OCT) for retinal nerve fiber layer (RNFL) thickness, and anterior segment OCT for bleb morphology, were all done pre- and postoperatively. Primary outcome measures were comparing preoperative to postoperative measurements and also comparing these measurements between SOAG and SACG. All patients were examined up to 1 year.

Results When preoperative IOP was compared with postoperative IOP, in each group, there was a statistically significant difference $(P<0.001)$. IOP percentage difference was statistically insignificantly different between both groups except at 1 month. According to Moorfields bleb grading system; postoperative bleb was better than the bleb of the previously failed trabeculectomy $(P<0.001)$, and there was a significant difference between group $A$ and $B$ regarding bleb area. Total success rate was $100 \%$; in group $\mathrm{A}$, complete success was
\end{abstract}

$100 \%$, while in group B it was $72.7 \%$

$(P=0.016)$.

Conclusion Our results suggest that Ologen may be a useful alternative to MMC in repeat trabeculectomy.

Eye (2016) 30, 1126-1134; doi:10.1038/eye.2016.114; published online 3 June 2016

\section{Introduction}

Trabeculectomy is the most commonly used surgery for lowering intraocular pressure (IOP) by providing a passage to the subconjunctival space. However, the success rate of this surgery has been limited by postoperative fibroblast proliferation and scarring of the filtering bleb. ${ }^{1}$ Numerous adjunctive modifications, such as antimetabolites, ${ }^{2}$ b-radiation, ${ }^{3}$ and anti-VEGF agents, ${ }^{4}$ have been developed to prevent scarring.

High-risk factors that lead to the failure of trabeculectomy include previous ocular surgery, specific types of glaucoma, for example, secondary glaucoma such as neovascular, uveitic, post-traumatic, and lens-induced glaucoma. ${ }^{5}$

Ologen is a biodegradable, porous, porcine, collagen implant. It influences the fibrosis process by guiding the patterns of fibroblast migration and normalizing secreted extracelluar matrix deposition. It also helps to separate the conjunctiva and episcleral surface preventing adhesions between them. ${ }^{6}$

Most of the studies have compared between ologen and mitomycin C (MMC) in primary glaucoma or in primary and secondary glaucomas collectively. Also, studies stated that there was no difference in the success rate between trabeculectomy in cases of open angle and angle closure glaucoma. ${ }^{7}$ So, in this work, 
we aimed to assess the IOP-lowering effect of trabeculectomy with ologen implant in cases of refractory secondary glaucoma following failed trabeculectomy with MMC as well as to compare its surgical outcome between open angle and angle closure cases.

The interesting points in our study were; is trabeculectomy with ologen implant effective in lowering IOP as the well established effect of trabeculectomy with MMC?, is there a difference in IOP reduction between the open angle and the angle closure glaucoma?, can ologen be a better alternative than MMC as a wound modulating agent in glaucoma surgeries?

\section{Materials and methods}

Approval for the study was obtained from the hospital's Ophthalmology Department Ethical Committee

(according to the WMA Declaration of Helsinki Principle). All patients received a thorough explanation of the study design and aims, and were provided with written informed consent.

This is a prospective interventional comparative study conducted on 40 eyes of 40 patients with medically uncontrolled secondary glaucoma, following previously failed trabeculectomy with MMC (0.4 mg/cc for $2 \mathrm{~min})$, and three attempts of needling with 1 month apart. Trabeculectomy with ologen implant was done 1 month after the last needling procedure.

All eyes had IOP above $21 \mathrm{mmHg}$ with the maximally tolerated anti-glaucoma medications (either due to side effects caused by adding more medications or due to extra cost of medications), glaucomatous visual field defects confirmed by 24-2 SITA Standard Humphrey visual field analysis and glaucomatous optic cupping. The study was performed during the time period from July 2013 to July 2014.

All eligible patients from Kasr Al-Ainy Cairo University Hospitals Ophthalmology Department Glaucoma outpatient clinic entered the study consecutively. Kasr Al-Ainy hospital is a central university hospital in Cairo and it is the center for referral from most of the governorates in Egypt.

The patients were divided into two groups; group A: 18 eyes of 18 patients with secondary open angle glaucoma (SOAG), and group B: 22 eyes of 22 patients with secondary angle closure glaucoma (SACG). SOAG is defined as glaucoma with open anterior chamber angle on gonioscopy, in the presence of an identifiable cause resulting in elevation of the IOP, while SACG is defined as the presence of an occludable angle on gonioscopy (posterior trabecular meshwork not seen in at least $180^{\circ}$ of the total circumference of the angle in primary position), with an identifiable cause leading to angle closure and elevation of IOP.
Two eyes with media opacities interfering with visual field testing and optical coherence tomography (OCT) imaging were excluded from the study; one eye had corneal opacities and the other had dense cataract requiring combined phacotrabeculectomy with ologen implant. Furthermore, one eye was excluded because the patient had not completed the 12 months of follow-up, and one more because the patient had undergone prior retinal segment surgery. None of the patients had previous anterior segment surgery except for the previous failed trabeculectomy. None of the eyes developed postoperative cataract following trabeculectomy with ologen implant. In all eyes with neovascular glaucoma, the retinal condition was treated and stabilized before the previous failed trabeculectomy by panretinal photocoagulation and nothing was needed regarding the retinal condition.

\section{Preoperative evaluation}

All the patients received complete ophthalmological examination, including; measurement of the bestcorrected visual acuity (BCVA), slit lamp examination (studying the old scleral bleb according to Moorfields bleb grading system), IOP measurement with Goldmann's applanation tonometry, dilated fundus examination, and gonioscopy for angle grading using Schaffer's method. The number of anti-glaucoma medications was recorded.

Humphrey SITA standard perimetry (Central 24-2 standard strategy) to assess the field of vision was done. Also spectral domain OCT was done using RTVue Fourier-Domain OCT (v 6.11.0.12, Optovue, Inc., USA) to assess retinal nerve fiber layer (RNFL) thickness. Also, anterior segment OCT (RTVue Fourier-Domain OCT, v 6.11.0.12, Optovue, Inc.) was used to study the old bleb morphology and for confirmation of the type of glaucoma. AS-OCT was done in down gaze to obtain maximal cross sectional exposure. Using a raster scan, AS-OCT (Anterior segment mode using the CAM-L) optical section was obtained that passed through the highest elevation on the surface of the bleb.

\section{Surgical technique}

All eyes underwent trabeculectomy with insertion of ologen implant, under peribulbar anesthesia. A fornixbased conjunctival incision was done. A rectangular $(4 \times 3 \mathrm{~mm})$ superficial scleral flap half of the sclera thickness was fashioned. Paracentesis for gradual decompression was followed by excision of trabecular meshwork block $(2 \times 1 \mathrm{~mm})$ by Kelly punch, peripheral iridectomy then suturing of the scleral flap using two 
10/0 nylon sutures at its corners. The scleral sutures were placed with minimal tension aiming for filtration on minimal pressure and a formed AC at the end of surgery. Ologen $(6 \mathrm{~mm}(\mathrm{D}) \times 2 \mathrm{~mm}(\mathrm{H}))$ was inserted between the posterior sclera and tenon's capsule, resting over the scleral flap. Finally the conjunctiva was sutured with water-tight $10 / 0$ nylon sutures.

Postoperatively, patients were prescribed topical combined antibiotics and steroids eye drops and were instructed for gradual tapering over six weeks. Postoperative cycloplegia was prescribed only when needed.

None of the eyes in either group needed suture lysis, needling with 5FU nor injections of 5FU, and none of the eyes with SOAG needed ALT or SLT.

\section{Postoperative follow-up}

All the patients were examined on the first day postoperatively, after 1 week, after 1 month, 6 months, then at 1 year postoperatively. Examination involved measurement of the BCVA, slit lamp examination (for clinical bleb morphology according to Moorfields bleb grading system and postoperative complications), and IOP measurement with Goldmann's applanation tonometry. In addition, Humphrey SITA standard perimetry and OCT (both retinal and anterior segment) were done at 6 months post operatively. Anterior segment OCT was done 6 months postoperatively as the ologen implant was expected to biodegrade after 3-6 months. ${ }^{8}$ Also, the number of antiglaucoma medications and the percentage change in the number of medications were recorded.

Surgical success rate was assessed. Complete success was defined as $5 \mathrm{mmHg}>\mathrm{IOP}<21 \mathrm{mmHg}$ and a relative decrease of $\geq 20 \%$ compared with the preoperative IOP, without any additional glaucoma surgery or antiglaucoma medication through all follow-up period. Qualified success was defined as $5 \mathrm{mmHg}>\mathrm{IOP}<21 \mathrm{mmHg}$ and an additional reduction of $\geq 20 \%$ in IOP compared with the preoperative IOP, without any additional glaucoma surgery, but with topical medications. Failure was defined as IOP $\geq 22 \mathrm{mmHg}$ despite maximal medication or IOP $\leq 5 \mathrm{mmHg}$ (on two or more examinations) with hypotony maculopathy or if an additional procedure like needling or repeat trabeculectomy was required to control the IOP or if there was loss of light perception.

Eyes were also assessed according to secondary success rate; number (and percentage) of eyes with IOP $<15 \mathrm{mmHg}$, those $15 \mathrm{mmHg} \geq \mathrm{IOP}<18 \mathrm{mmHg}$ and those $18 \mathrm{mmHg} \geq$ IOP $<21 \mathrm{mmHg}$.

\section{Statistical analysis}

Data were statistically described in terms of range, mean $\pm S D$, median frequencies (number of cases), and percentages when appropriate. Comparison of numerical variables between the study groups was done using Mann-Whitney $U$-test for independent samples, while within group comparison of numerical variables was performed using the Wilcoxon SignedRank test. For comparing categorical data, $\chi^{2}$-test was performed. All $P$-values $<0.05$ was considered statistically significant. Complete success was analyzed using Kaplan-Meier survival analysis. Predictors of incident glaucoma were examined using multiple linear regression analysis to study their effect on postoperative glaucoma. All statistical calculations were done using computer programs Microsoft Excel 2007 (Microsoft Corporation, NY, USA) and SPSS (Statistical Package for the Social Science; SPSS Inc., Chicago, IL, USA) version 18 for the Microsoft Windows. Due to lack of any reports for the Egyptian population, sample size (16 eyes for each group) was calculated using MedCalc 10.2.0.0, by referring to success rates from literature.

\section{Results}

Our study was performed on 40 eyes (40 patients) suffering from SOAG and SACG, requiring glaucoma surgery due to previously failed trabeculectomy.

\section{Patient data}

In group $\mathrm{A}$, the patients' mean age was $48.44 \pm 3.48$ years (with 12 males), while in group $B$, it was $54.73 \pm 2.06$ years (with 10 males). Type of glaucoma in each group is shown in Table 1.

All the eyes with angle closure (group B) suffered from synechial angle closure in all quadrants; grade 0 according to Shaffer's classification as detected by indentation gonioscopy using Ziess 4 mirror goniolens.

Preoperative and postoperative BCVA is shown in Table 1. There was no significant difference between preoperative and postoperative $\operatorname{BCVA}(P=1.00)$ in each group.

The preoperative and postoperative anti-glaucoma medication score is shown in Table 1. Preoperative medication score compared to postoperative score, in each group was statistically significant $(P<0.001)$. The percentage difference between preoperative and postoperative medication score was compared between both groups and was statistically significant $(P=0.018)$. 
Table 1 Demographic data, preoperative and postoperative best-corrected visual acuity (BCVA) and anti-glaucoma medication score, and type of glaucoma in both groups

\begin{tabular}{|c|c|c|c|}
\hline & Group A & Group B & P-value \\
\hline Age in years (range $($ mean $\pm S D)$ ) & $15-65(48.44 \pm 3.48)$ & $34-69(54.73 \pm 2.06)$ & 0.156 \\
\hline Gender (males) (No. (\%)) & $12(66.7 \%)$ & $10(45.5 \%)$ & 0.180 \\
\hline Preoperative BCVA LogMAR (range (mean \pm SD)) & $0.8-0.1(0.3 \pm 0.0)$ & $0.4-0.1(0.2 \pm 0.0)$ & 0.906 \\
\hline Postoperative BCVA LogMAR (range (mean \pm SD)) & $0.8-0.1(0.3 \pm 0.0)$ & $0.4-0.1(0.2 \pm 0.0)$ & 0.906 \\
\hline \multicolumn{4}{|l|}{ Preoperative medscore (No. (\%)) } \\
\hline 0 & $0 \%$ & $0 \%$ & \multirow[t]{4}{*}{0.002} \\
\hline 1 & $4(22.2 \%)$ & $0 \%$ & \\
\hline 2 & $14(77.8 \%)$ & $16(72.7 \%)$ & \\
\hline 3 & $0 \%$ & $6(27.3 \%)$ & \\
\hline \multicolumn{4}{|l|}{ Postoperative medscore (No. (\%)) } \\
\hline 0 & $18(100 \%)$ & $16(72.7 \%)$ & \multirow[t]{4}{*}{0.024} \\
\hline 1 & $0 \%$ & $6(27.3 \%)$ & \\
\hline 2 & $0 \%$ & $0 \%$ & \\
\hline 3 & $0 \%$ & $0 \%$ & \\
\hline \multirow[t]{3}{*}{ Type of glaucoma (No. (\%)) } & \multirow{3}{*}{$\begin{array}{c}\text { Angle recession: } 6(33.3 \%) \\
\text { Pseudoexfoliation: } 12(66.7 \%)\end{array}$} & Neovascular: 4 (18.2\%) & \\
\hline & & Inflammatory: $6(27.3 \%)$ & \\
\hline & & Traumatic: 12 (54.5\%) & \\
\hline
\end{tabular}

$P$-value $<0.05$ is considered statistically significant. Bold and italic values are statistically significant.

\section{Bleb morphology}

Preoperatively, all eyes showed cystic fibrotic scleral bleb due to previously failed trabeculectomy with MMC, while postoperatively they all had new diffuse vascular blebs, as seen clinically $(P<0.001$ regarding central and peripheral areas, height and vascularity, according to Moorfields bleb grading system) and by the anterior segment OCT (AS-OCT) at 6 months postoperatively (Figure 1a).

There was a significant difference between group A and $B$ regarding postoperative bleb central and peripheral areas $(P=0.018$, and $P=0.001$, respectively; Table 2).

\section{Intraocular pressure}

The preoperative and postoperative IOP measurements at different postoperative visits, the drop and percentage drop in IOP are all shown in Table 3.

IOP changes in group A were compared with those in group B using Mann-Whitney test. IOP percentage difference was statistically insignificantly different between both groups except at 1 month. (Table 3)

Preoperative IOP was compared with postoperative IOP at 1 day, 1 week, 1 month, 6 months, and 1 year, in each group, and was found to be statistically significant $(P<0.001$ at each follow-up period) using Wilcoxon Signed-Rank test (Figure 1b).

In group $\mathrm{B}$, six eyes developed IOP $>21 \mathrm{mmHg}$; two of them at 3 months and four eyes at 2 months postoperatively. They were all controlled with one antiglaucoma medication. Using multiple linear regression analysis, preoperative medication score $(P=0.030)$ was a significant risk factor for postoperative glaucoma in group $\mathrm{B}$, while preoperative IOP $(P=0.379)$ and type of SACG $(P=0.474)$ were not.

\section{Success rate}

In group $\mathrm{A}$, all the eyes achieved complete success $(100 \%)$, while in group B complete success was $72.7 \%$ $(P=0.016)$. None of the eyes developed hypotony. Also the eyes were graded according to secondary success; IOP $<15 \mathrm{~mm} \mathrm{Hg}, 15 \mathrm{mmHg} \geq \mathrm{IOP}<18 \mathrm{mmHg}$ and $18 \mathrm{mmHg} \geq \mathrm{IOP}<21 \mathrm{mmHg}$ (Table 4; Figures 1c and d).

\section{Visual field changes}

Preoperative and postoperative mean deviation (MD) and pattern standard deviation (PSD) and changes in these parameters were recorded (Table 3).

Comparing group A with group B using MannWhitney test was statistically insignificant except for preoperative PSD (Table 3).

Also comparing preoperative to postoperative MD and PSD in group A, using Wilcoxon Signed-Rank test, was statistically insignificant $(P=0.085$ and $P=0.500$, respectively); while in group $B, M D$ significantly differed postoperatively $(P<0.001)$ but PSD did not $(P=0.844$; Figures $1 \mathrm{e}$ and $\mathrm{f}$ ). 
a
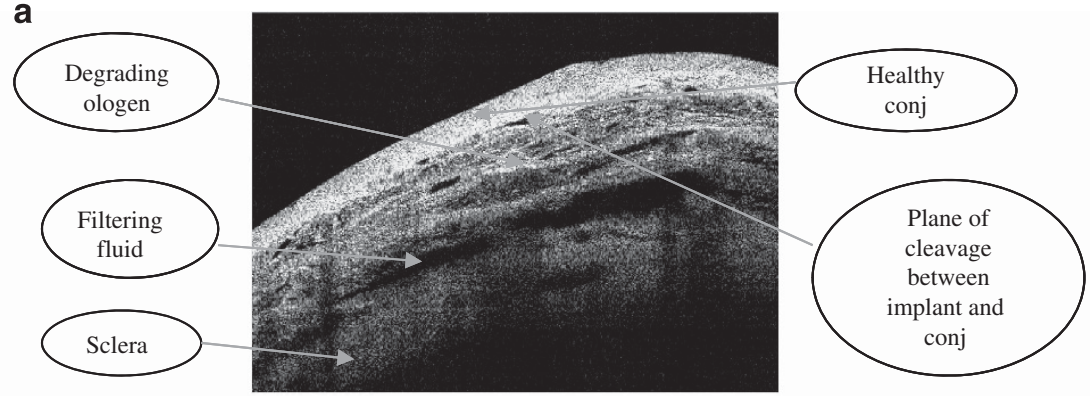

b

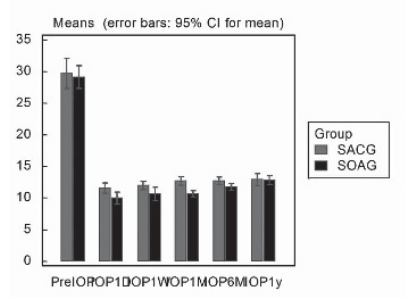

e

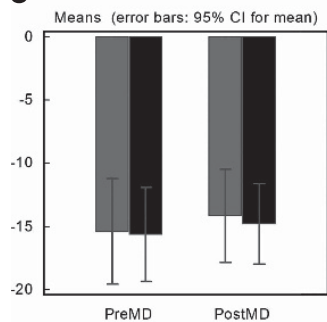

C

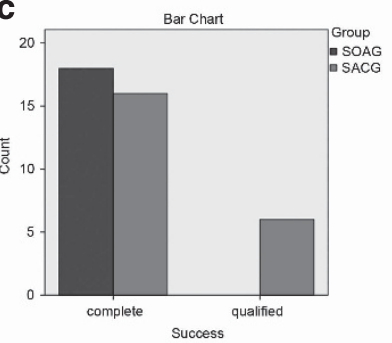

f

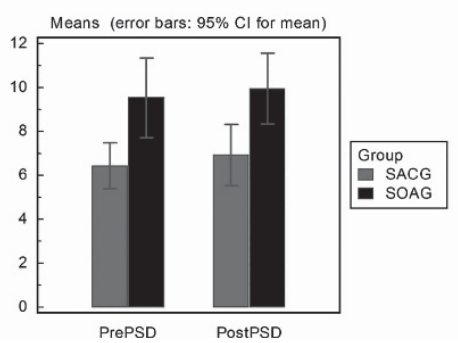

d

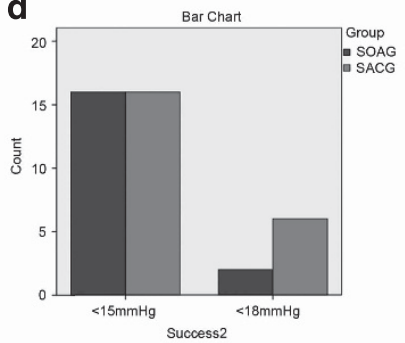

9 Means (error bars: $95 \% \mathrm{Cl}$ for mean)

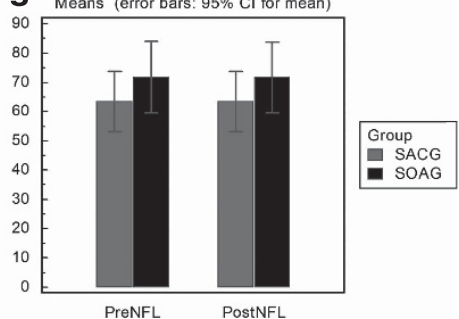

Figure 1 (a) Anterior segment OCT for the ologen bleb after 6 months showing clear plane of cleavage between the implant and the conjunctiva, healthy non scarred overlaying conjunctiva, clear filtering fluid under the conjunctiva. (b) Comparing pre- to postoperative IOP at different postoperative visits in each group. (c) Comparing primary success between groups A and B. (d) Comparing secondary success between groups A and B. (e) Comparing pre- to postoperative MD in each group. (f) Comparing pre- to postoperative PSD in each group. (g) Comparing pre- to postoperative RNFL thickness in each group.

\section{Optical coherence tomography}

RNFL thickness was assessed pre- and postoperatively in each group. (Table 3; Figure 1g).

Postoperative RNFL thickness did not differ significantly from that preoperatively in group $\mathrm{A}$ or $\mathrm{B}$ ( $P=0.743, P=0.407$, respectively).

\section{Complications}

None of the eyes developed intra- or postoperative complications except for one eye which developed a dellen.

\section{Discussion}

Trabeculectomy differs from most surgical procedures, as inhibition of wound healing is desirable to achieve surgical success. ${ }^{9}$ However, scarring at the episcleral and deep Tenon's level is the main cause of failure of trabeculectomy. Several factors influence and control the complicated mechanism of wound healing. Extracellular matrix components such as collagen and fibronectin, cell adhesion molecules such as selectins and integrins, and different growth factors are intimately involved with the fibroblasts. ${ }^{10}$

Several surgical and pharmacologic techniques have been introduced to enhance the success in eyes with poor surgical prognoses. But, no effective and safe agent has been identified that can inhibit fibrosis, without complications, in the glaucoma filtering wound created by trabeculectomy. Although antimetabolites have revolutionized glaucoma surgery, the use of these agents is still associated with substantial risk. ${ }^{11}$

Up to our knowledge, this was the first work to highlight the use of ologen implant in cases of secondary glaucoma separately (not in conjunction with primary glaucoma), which represents one of the important risk 
Table 2 Preoperative (of previous failed trabeculectomy) and postoperative bleb morphology in both groups according to Moorfield's Bleb Grading System

\begin{tabular}{|c|c|c|c|c|}
\hline Bleb & Score & Group A (number of eyes (\%)) & Group B (number of eyes (\%)) & P-value \\
\hline \multicolumn{5}{|l|}{ Preoperative } \\
\hline \multirow[t]{2}{*}{ Central area } & $2(25 \%)$ & $16(88.9 \%)$ & $20(90.9 \%)$ & 0.832 \\
\hline & $3(50 \%)$ & $2(11.1 \%)$ & $2(9.1 \%)$ & \\
\hline \multirow[t]{2}{*}{ Peripheral Area } & $2(25 \%)$ & $16(88.9 \%)$ & $20(90.9 \%)$ & 0.832 \\
\hline & $3(50 \%)$ & $2(11.1 \%)$ & $2(9.1 \%)$ & \\
\hline \multirow[t]{2}{*}{ Height } & 1 & $12(66.7 \%)$ & $12(54.5 \%)$ & 0.436 \\
\hline & 2 & $6(33.3 \%)$ & $10(45.5 \%)$ & \\
\hline Vascularity & 1 (Avascular) & $18(100 \%)$ & $22(100 \%)$ & \\
\hline Subconjunctival blood & & $0 \%$ & $0 \%$ & \\
\hline \multicolumn{5}{|l|}{ Postoperative } \\
\hline \multirow[t]{2}{*}{ Central area } & $3(50 \%)$ & $2(11.1 \%)$ & $10(45.5 \%)$ & 0.018 \\
\hline & $4(75 \%)$ & $16(88.9 \%)$ & $12(54.5 \%)$ & \\
\hline \multirow[t]{2}{*}{ Peripheral Area } & $3(50 \%)$ & $4(22.2 \%)$ & $16(72.7 \%)$ & 0.001 \\
\hline & $4(75 \%)$ & $14(77.8 \%)$ & $6(27.3 \%)$ & \\
\hline \multirow[t]{2}{*}{ Height } & 2 & $8(44.4 \%)$ & $6(27.3 \%)$ & 0.257 \\
\hline & 3 & $10(55.6 \%)$ & $16(72.7 \%)$ & \\
\hline \multirow[t]{2}{*}{ Vascularity } & 2 (Normal) & $16(88.9 \%)$ & $18(81.8 \%)$ & 0.533 \\
\hline & 3 (Mild) & $2(11.1 \%)$ & $4(18.2 \%)$ & \\
\hline Subconjunctival blood & & $0 \%$ & $0 \%$ & \\
\hline
\end{tabular}

$P$-value $<0.05$ is considered statistically significant. Bold and italic values are statistically significant.

factors for failure of trabeculectomy. In this work, we also compared the surgical outcome between open angle (SOAG) and angle closure secondary glaucoma (SACG).

Many comparative controlled trials have compared the efficacy and tolerability of trabeculectomy augmented with Ologen versus trabeculectomy plus MMC. Some studies have found that the techniques were comparable in IOP-lowering efficacy. ${ }^{12-15}$ Our challenge was to know if the ologen could be better than MMC as a wound modulating agent so nothing could prove this except to put the ologen implant after trabeculectomy in the same eye where the MMC was not efficient.

In a study by Johnson and Sarkisian, ${ }^{16}$ they compared outcomes between patients undergoing trabeculectomy with an Ex-PRESS mini glaucoma device using MMC to those undergoing the same procedure using a subconjunctival Ologen, in 99 eyes collectively having primary and secondary open angle and angle closure glaucoma. There was no statistically significant difference between either group for the rates of achieving a specified postoperative IOP either with (qualified success) or without medications. There was no statistically significant difference between the 2 groups in the mean number of postoperative medications required. Both groups had similar rates of complications. ${ }^{16}$

In 2015, Ji et al ${ }^{17}$ conducted a meta-analysis of randomized clinical trials to assess the efficacy and safety of trabeculectomy with Ologen implant versus trabeculectomy with MMC for treatment of glaucoma. A total of 6 studies including 224 participants were included in this meta-analysis. Ologen implant was associated with a numerically lower but non-significant percentage reduction in IOP compared with MMC. There was no significant difference in the reduction in glaucoma medications, success rate, and incidence of complications. ${ }^{17}$

Also, Dada et al ${ }^{18}$ evaluated the outcomes of trabeculectomy with use of a subconjunctival Ologen combined with MMC in 33 eyes with primary open angle glaucoma. They declared that it appears to offer encouraging short-term results for IOP control. ${ }^{18}$ In a similar study, Min et al ${ }^{19}$ studied surgical outcomes and wound healing reaction of a filtering bleb after trabeculectomy using MMC-soaked ologen. They found that it did not exert any synergistic effect in terms of a reduction in IOP, but it resulted in comparatively stable IOP and did not aggravate wound healing or scar formation, while encapsulated blebs were more common and developed more rapidly. ${ }^{19}$

In our work, the preoperative IOP was insignificantly different between SOAG (group A) and SACG (group B) glaucoma, while postoperative IOP was significantly lower in each group at each follow up period $(P<0.001)$. The percentage drop in IOP in group A was statistically insignificantly different from that in group B at all follow up periods except at 1 month postoperative when group A percentage drop was significantly more than group $B$ $(P=0.006)$.

Total success rate (complete+qualified) in our study was $100 \%$ in each group. All the eyes $(100 \%)$ in group A 
Table 3 The preoperative and postoperative intraocular pressure (IOP) measures at different postoperative visits, the drop and percentage drop in IOP, field changes (Mean deviation (MD) and pattern standard deviation (PSD)) and retinal nerve fiber layer (RNFL) changes in each group

\begin{tabular}{|c|c|c|c|c|c|}
\hline & \multicolumn{2}{|c|}{ Group A } & \multicolumn{2}{|c|}{ Group B } & \multirow[t]{2}{*}{ P-value } \\
\hline & Mean $\pm S D$ & Range & Mean $\pm S D$ & Range & \\
\hline \multicolumn{6}{|l|}{$\mathrm{IOP}(\mathrm{mmHg})$} \\
\hline Preoperative & $29.11 \pm 0.85$ & $24-34$ & 29.73 & $22-38$ & 0.701 \\
\hline \multicolumn{6}{|l|}{1 day postoperative } \\
\hline Value & $10.0 \pm 0.46$ & $6-12$ & $11.54 \pm 0.41$ & $8-14$ & \\
\hline Drop & $19.11 \pm 0.97$ & $12-24$ & $18.18 \pm 1.19$ & $11-28$ & \\
\hline \% drop & $65.11 \pm 1.98$ & $50-79.31$ & $60.05 \pm 1.97$ & $46.15-73.68$ & 0.102 \\
\hline \multicolumn{6}{|c|}{1 week postoperative } \\
\hline Value & $10.67 \pm 0.51$ & $6-14$ & $12.0 \pm 0.32$ & $10-14$ & \\
\hline Drop & $18.44 \pm 0.997$ & $13-24$ & $17.73 \pm 0.95$ & $12-24$ & \\
\hline \% drop & $62.80 \pm 2.08$ & $52-79.31$ & $58.997 \pm 1.13$ & $50-66.67$ & 0.275 \\
\hline \multicolumn{6}{|c|}{1 month postoperative } \\
\hline Value & $10.67 \pm 0.23$ & $10-12$ & $12.73 \pm 0.34$ & $10-16$ & \\
\hline Drop & $18.44 \pm 0.94$ & $13-24$ & $17.0 \pm 0.97$ & $10-24$ & \\
\hline \% drop & $62.70 \pm 1.54$ & $52-70.59$ & $56.37 \pm 1.42$ & $45.45-66.67$ & 0.006 \\
\hline \multicolumn{6}{|c|}{6 months postoperative } \\
\hline Value & $11.78 \pm 0.27$ & $10-14$ & $12.73 \pm 0.28$ & $12-16$ & \\
\hline Drop & $17.33 \pm 0.94$ & $12-22$ & $17.0 \pm 1.08$ & $10-24$ & \\
\hline \% drop & $58.85 \pm 1.73$ & $46.15-66.67$ & $56.07 \pm 1.64$ & $46.15-66.67$ & 0.325 \\
\hline \multicolumn{6}{|l|}{1 year postoperative } \\
\hline Value & $12.89 \pm 0.33$ & $12-16$ & $12.91 \pm 0.47$ & $10-16$ & \\
\hline Drop & $16.22 \pm 0.83$ & $11-22$ & $16.82 \pm 1.29$ & $10-25$ & \\
\hline$\%$ drop & $55.21 \pm 1.495$ & $44-64.71$ & $55.02 \pm 2.47$ & $38.46-71.43$ & 0.913 \\
\hline \multicolumn{6}{|l|}{ Field } \\
\hline \multicolumn{6}{|l|}{$M D(d B)$} \\
\hline Preoperative & $-15.62 \pm 1.76$ & -4.56 to -28.10 & $-15.38 \pm 2.00$ & -2.03 to -29.63 & 0.957 \\
\hline Postoperative & $-14.78 \pm 1.50$ & -4.33 to -22.98 & $-14.15 \pm 1.77$ & -1.5 to -23.79 & \\
\hline Change & $0.84 \pm 0.49$ & -0.56 to 6.42 & $1.23 \pm 0.38$ & -0.03 to 6.21 & \\
\hline \% Change & $3.49 \pm 1.82$ & $0.4-22.85$ & $7.43 \pm 1.85$ & $0.41-26.11$ & 0.114 \\
\hline \multicolumn{6}{|l|}{$P S D(d B)$} \\
\hline Preoperative & $9.53 \pm 0.86$ & $3.2-15.6$ & $6.43 \pm 0.51$ & $1.57-9.82$ & 0.003 \\
\hline Postoperative & $9.95 \pm 0.76$ & 3.3-15.3 & $6.92 \pm 0.67$ & 1.5-11.81 & \\
\hline Change & $0.42 \pm 0.31$ & -0.3 to 3.97 & $0.496 \pm 0.38$ & -1.06 to 4.11 & \\
\hline$\%$ Change & $8.70 \pm 5.89$ & $1.92-77.24$ & $6.38 \pm 5.33$ & 0-60.18 & 0.072 \\
\hline \multicolumn{6}{|l|}{ OCT } \\
\hline \multicolumn{6}{|l|}{ RNFL $(\mu)$} \\
\hline Preoperative & $71.63 \pm 5.78$ & $41.96-112$ & $63.42 \pm 4.97$ & $45.45-114.6$ & 0.211 \\
\hline Postoperative & $71.61 \pm 5.77$ & $40.2-114.3$ & $63.47 \pm 4.91$ & $45.43-113.9$ & \\
\hline Change & $-0.03 \pm 0.199$ & $-8.96-7.77$ & $0.05 \pm 0.21$ & $-2.51-1.12$ & \\
\hline$\%$ Change & $0.13 \pm 2.06$ & $0.1-18.23$ & $0.21 \pm 0.39$ & $0.01-4.61$ & 0.549 \\
\hline
\end{tabular}

$P$-value $<0.05$ is considered statistically significant. Bold and italic values are statistically significant.

achieved complete success, while $72.7 \%$ in group B achieved complete success, with significant difference between both $(P=0.016)$, as six eyes in group B had their $\mathrm{IOP}>21 \mathrm{mmHg}$ and were controlled with one antiglaucoma medication, which was significantly more than group A $(P=0.024)$. None of the eyes developed hypotony. Regarding secondary success rate, there was no significant difference between both groups $(P=0.204)$. Preoperative medication score was a significant risk factor for postoperative glaucoma, which might explain higher success rate in OAG compared to ACG.
Regarding visual field changes, percentage change in MD and PSD were insignificantly different between both groups. Comparing preoperative to postoperative MD and PSD was statistically insignificant, except for MD in group B which significantly differed postoperatively $(P<0.001)$. RNFL thickness was insignificantly different postoperatively and so was the percentage change in thickness between both groups.

We also studied the filtering scleral bleb in each group clinically and using anterior segment OCT. According to Moorfields bleb grading system; there was a significant 
Table 4 Primary and secondary success rates in both groups

\begin{tabular}{|c|c|c|c|c|}
\hline & & Group $A$ & Group B & P-value \\
\hline \multicolumn{5}{|l|}{ Primary success } \\
\hline Complete & Count (\%) & $18(100.0 \%)$ & $16(72.7 \%)$ & 0.016 \\
\hline Qualified & Count (\%) & $0 \%$ & $6(27.3 \%)$ & \\
\hline Failure & Count $(\%)$ & $0 \%$ & $0 \%$ & \\
\hline \multicolumn{5}{|l|}{ Secondary success } \\
\hline$<15 \mathrm{mmHg}$ & Count (\%) & $16(88.9 \%)$ & $16(72.7 \%)$ & 0.204 \\
\hline $15 \mathrm{mmHg} \geq \mathrm{IOP}<18 \mathrm{mmHg}$ & Count (\%) & $2(11.1 \%)$ & $6(27.3 \%)$ & \\
\hline $18 \mathrm{mmHg} \geq \mathrm{IOP}<21 \mathrm{mmHg}$ & Count (\%) & $0 \%$ & $0 \%$ & \\
\hline
\end{tabular}

$P$-value $<0.05$ is considered statistically significant. Bold and italic values are statistically significant.

difference between group A and B regarding postoperative bleb central and peripheral areas, and a significant difference between preoperative (due to previous failed trabeculectomy) and postoperative bleb morphology in each group. AS-OCT showed that ologen was still present in $100 \%$ of the eyes at 6 months, while in a study by Boey et $a l,{ }^{20}$ it was seen in AS-OCT images in $39.4 \%$ of the eyes at 90 days.

In our work, we did not take releasable sutures and also laser suture lysis was not needed and this is one of the advantages of the ologen implant as the scleral sutures could be left not too tight and putting the ologen implant on the posterior edge of the flap can press against the flap. The implant influences the aqueous flow by maintaining pressure on top of the sclera flap and by acting as a reservoir as the aqueous humor is absorbed into its porous structure thus preventing the hypotony and at the same time avoiding the need for removing the sutures. $^{20}$

A review of prior studies on the efficacy of Ologen reveals mixed results. Although studies have shown that blebs following the use of Ologen tend to be more vascular and low-lying, there may be a concern that this clinically robust tissue fails to achieve the same IOPlowering effect as the avascular tissue after MMC. ${ }^{21}$

In this study, the postoperative medication score differed significantly from the preoperative in each group $(P<0.001)$, and the percentage difference in medication score differed significantly between both groups $(P=0.018)$.

None of our eyes developed significant complications except for one eye that developed a dellen, due to conjunctival elevation caused by a slightly anteriorly placed ologen. In the study by Dada et al, ${ }^{18}$ there were no sight-threatening complications; however, two eyes developed hypotony during the early postoperative course due to leaky conjunctival wound. Another case was noted to have implant exposure at 1 week. Early conjunctival leakage was also reported by Rosentreter et $a l^{21}$ in $30 \%$ of their cases undergoing trabeculectomy with Ologen implantation. This emphasizes that during surgery the conjunctiva should be carefully draped over the implant and closed meticulously to avoid postoperative wound leaks, and the implant should be placed at a slightly posterior position such that it does not impinge on the suture line at the limbus. In the study by Min et a ${ }^{19}$ encapsulated blebs occurred in 9 out of 30 eyes among which digital massage was performed on 8 eyes and needling was performed on 1 eye.

To conclude, Ologen may be a useful alternative to MMC in repeat trabeculectomy.

Limitations to our work include; the higher preoperative medication score for ACG which might explain higher success rate with OAG. Also, it was difficult to keep the observers of IOP masked as eyes with SACG might have obvious PAS from the slit lamp evaluation.

\section{Summary}

What was known before

- Most of the studies have compared between ologen and mitomycin $\mathrm{C}(\mathrm{MMC})$ in primary glaucoma or in primary and secondary glaucomas collectively Also, studies stated that there was no difference in the success rate between trabeculectomy in cases of open angle and angle closure glaucoma.

What this study adds

- Our results suggest that Ologen may be a useful alternative to MMC in repeat trabeculectomy.

\section{Conflict of interest}

The authors declare no conflict of interest.

\section{References}

1 Picht G, Grehn F. Classification of filtering blebs in trabeculectomy: biomicroscopy and functionality. Curr Opin Ophthalmol 1998; 9: 2-8. 
2 Beckers HJ, Kinders KC, Webers CA. Five-year results of trabeculectomy with mitomycin C. Graefes Arch Clin Exp Ophthalmol 2003; 241: 106-110.

3 Kirwan JF, Cousens S, Venter L, Cook C, Stulting A, Roux P et al. Effect of beta radiation on success of glaucoma drainage surgery in South Africa: randomised controlled trial. BMJ 2006; 333: 942.

4 Akkan JU, Cilsim S. Role of subconjunctival bevacizumab as an adjuvant to primary trabeculectomy: a prospective randomized comparative 1-year follow-up study. J Glaucoma 2015; 24(1): 1-8.

5 Broadway DC, Chang LP. Trabeculectomy, risk factors for failure and the preoperative state of the conjunctiva. J Glaucoma 2001; 10(3): 237-249.

6 Hsu WC, Ritch R, Krupin T, Chen HS. Tissue bioengineering for surgical bleb defects: an animal study. Graefes Arch Clin Exp Ophthalmol 2008; 246: 709-717.

7 Sihota R, Gupta V, Agarwal HC. Long-term evaluation of trabeculectomy in primary open angle glaucoma and chronic primary angle closure glaucoma in an Asian population. Clin Experiment Ophthalmol 2004; 32(1): 23-28.

8 Zelefsky JR, Hsu WC, Ritch R. Biodegradable collagen matrix implant for trabeculectomy. Expert Rev Ophthalmol 2008; 3: 613-617.

9 Dvorak HF. Tumors: wounds that do not heal. Similarities between tumor stroma generation and wound healing. N Engl J Med 1986; 315(26): 1650-1659.

10 Broadway D, Grierson I, Hitchings R. The effect of topical anti-glaucomatous medications on the cell profile of the conjunctiva. Curr Opin Ophthalmol 1993; 4: 51-57.

11 Chen C. Enhanced intraocular pressure controlling effectiveness of trabeculectomy by local application of mitomycin C. Trans Asia Pac Acad Ophthalmol 1983; 9: 172-177.

12 Marey HM, Mandour SS, Ellakwa AF. Subscleral trabeculectomy with mitomycin-C versus ologen for treatment of glaucoma. J Ocul Pharmacol Ther 2013; 29 330-334.
13 Senthil S, Rao HL, Babu JG, Mandal AK, Garudadri CS. Comparison of outcomes of trabeculectomy with mitomycin C vs. ologen implant in primary glaucoma. Indian J Ophthalmol 2013; 61(7): 338-342.

14 Nilforushan N, Yadegari M, Hodjat P. Comparison of the success rate of trabeculectomy with OculusGen versus trabeculectomy with mitomycin C. Iran J Ophthalmol 2011; 23: 3-12.

15 Cillino S, Di Pace F, Cillino G, Casuccio A. Biodegradable collagen matrix implant vs mitomycin-C as an adjuvant in trabeculectomy: a 24-month, randomized clinical trial. Eye (Lond) 2011; 25: 1598-1606.

16 Johnson MS, Sarkisian SR Jr. Using a collagen matrix implant (Ologen) versus mitomycin-C as a wound healing modulator in trabeculectomy with the Ex-PRESS mini glaucoma device: a 12-month retrospective review. J Glaucoma 2014; 23: 649-652.

17 Ji Q, Qi B, Liu L, Guo X, Zhong J. Efficacy and safety of ologen implant versus mitomycin $\mathrm{C}$ in primary trabeculectomy: a meta-analysis of randomized clinical trials. J Glaucoma 2015; 24: e88-e94.

18 Dada T, Kusumesh R, Bali SJ, Sharma S, Sobti A, Arora V et al. Trabeculectomy with combined use of subconjunctival collagen implant and low-dose mitomycin C. J Glaucoma 2013; 22: 659-662.

19 Min JK, Kee CW, Sohn SW, Lee HJ, Woo JM, Yim JH. Surgical outcome of mitomycin C-soaked collagen matrix implant in trabeculectomy. J Glaucoma 2013; 22: 456-462.

20 Boey PY, Narayanaswamy A, Zheng C, Perera SA, Htoon $\mathrm{HM}$, Tun TA et al. Imaging of blebs after phacotrabeculectomy with Ologen collagen matrix implants. Br J Ophthalmol 2011; 95: 340-344.

21 Rosentreter A, Schild AM, Jordan JF, Krieglstein GK, Dietlein TS. A prospective randomized trial of trabeculectomy using mitomycin C vs an ologen implant in open angle glaucoma. Eye 2010; 24: 1449-1457. 